\section{Urinary Excretion of a Substance after a Single Dose}

AfTer a single dose of a substance, its urinary excretion is commonly highest during the subsequent twenty-four hours, and then decreases with time. It is often desirable to compare different urinary excretion-rates, to extrapolate or interpolate, etc., so that any method of plotting the data such that the relationship is linear between some functions of urinary excretion and time has obvious advantages.

During some work on the metabolism of radioactive phosphorus after oral administration of a single dose, we have found that the cumulative excretion of phosphorus-32 is linearly related to the logarithm of the time. Line $A$ of Fig. I is a typical example of our findings.

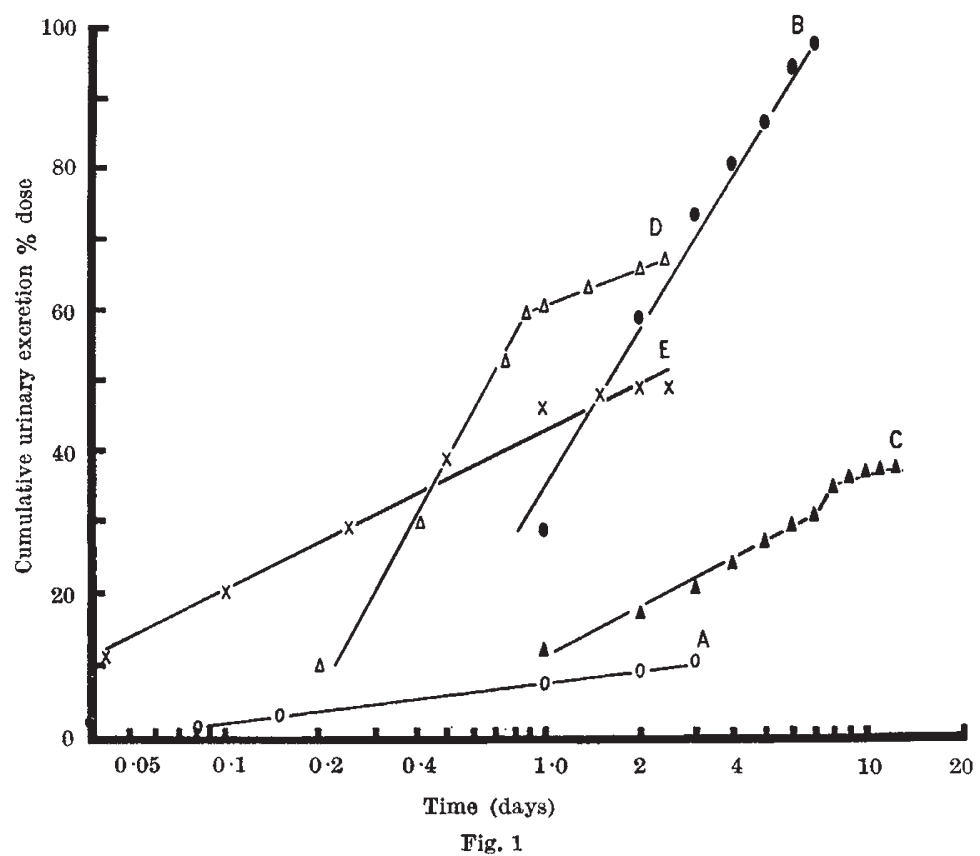

This linear relationship appears to be a general one. Thus, line $B$ is derived from some results reported by Van Der Werff ${ }^{1}$, after the subcutaneous injection of bismuth-206 in the form of bismuth citrate. Line $C$ shows another set of results re-plotted from the same paper, this time when the bismuth was mixed with a charcoal suspension and given by intravenous injection. This line shows a discontinuity, which may possibly be due to instability of the bismuth-charcoal suspension.

Wilkinson and Storey ${ }^{2}$ have published some cumulative urinary excretion figures for polyvidone, a plasma substitute which is not metabolized. Line $D$ shows the results from one of their cases, when plotted on a logarithmic time-scale. This line shows an abrupt change in slope which was not obvious when the data were plotted on a linear time-scale.

We have found this linear relationship very useful in our work on phosphorus-32, and we have expressed the slope of the line as the 'double time factor'. This is defined as the quantity $Q$ excreted in the urine, such that if a quantity $x$ is excreted in time $t$, a quantity $x+Q$ is excreted in time $2 t$. It is numerically equal to the quantity excreted during the second day. It can be shown that:

$$
x \log 2=Q \log \frac{t}{t_{0}},
$$

where $t_{0}$ is the intercept of the line on the time axis. Differentiating, we have

$$
\frac{\mathrm{d} x}{\mathrm{~d} t}=1 \cdot 44 \frac{Q}{t} .
$$

Thus, the urinary excretion-rates may be expressed in terms of one parameter, the double time factor.

This method of plotting the urinary excretion has the further advantage that, where there are any discontinuities, these become obvious and precisely located in time.

It is not, however, the only possible way of endeavouring to fit cumulative excretion figures to an equation. For example, Haigh and Reiss ${ }^{3}$ quote the relationship :

$$
x=x_{\max }\{1-\exp (-\lambda t)\},
$$

where $\lambda$ defines the rate at which excretion proceeds, and $x_{\max }$. is the cumulative urinary excretion when this is complete. Their figures for the cumulative urinary excretion of iodine131, when plotted on a logarithmic time-scale, give the line $E$ of Fig. 1 .

Equations (1) and (3) are not mutually consistent, and a particular set of results may prove to be a more or less good fit for either. The form of equation (3) is more logically explained than that of equation (1), but in a few cases tested by both equations, in general, it did not lead to better fits. Equation (1) has the advantages that it is very simple to apply, and that its use $d$ es not necessitate an estimate of the value of $x_{\max }$. which appears in equation (3), and which may be unknown. On the other hand, equation (1) is a divergent function of $x$, and in some cases, for example line $B$, obviously cannot hold when $t$ becomes large.

Acknowledgments are due to the

British Empire Cancer Campaign for financial assistance.

Marie Curie Hospital,

BABETTE E. STERN

London, N.W.3.

Sept. 2.

${ }^{2}$ Van Der Werff, J. T., "Bismuth-206. A New Radioactive Isotope for Therany". Second Radioisotope Conference, Oxford (Butterworths Scientific Publications, London, 1954).

"Wilkinson, A. W., and Storey, I. D. E., Lancet, i, 1269 (1954).

${ }^{3}$ Haigh, C. P., and Reiss, M., Brit. J. Radiol., 23, 538 (1950).

\section{Action of Ribonuclease on Certain Ascites Tumours}

Previous papers ${ }^{1}$ have described the action of ribonuclease on the cells of the Ehrlich carcinoma in vitro, and the object of the present communication is briefly to record results obtained in vivo, following the administration of ribonuclease to animals bearing various ascites tumours. These experiments involved three hundred animals carrying the Ehrlich or Krebs mouse carcinomata, or the Walker rat carcinoma 256.

The mean survival-time of the experimental animals was determined in relation to the number of injected 